\title{
Effect of the Site of Fixation of Foley Catheter in the Cervix on the Collected Embryos in the Cow
}

\author{
Wafir Mahdi SALEH and Ihsan H. AL-TIMIMI
}

Department of Surgery and Obstetrics, College of Vet. Medicine. University of Baghdad. Baghdad-Iraq

\section{Summary}

Thirty six trials of flushing with riched Delbeco`s phosphate buffer media applied in this study using a plastic two ways folly catheter, introduced by metal robe inside the cervix of donor cows and by air bubble fixed it in the cervical canal in 3 positions, one near the cervicovaginal opening or at the first third of the cervical canal, the $2^{\text {nd }}$ one at the middle third of the cervical canal and the $3^{\text {rd }}$ one near the utero-cervical opening or at the last third of the cervical canal. The process of flushing was carried out after the fixation of the folly catheter in the three sites and the number of embryos collected by each collection site.

The results showed that the number of embryos detected in the third method where the fixation was in the last $3^{\text {rd }}$ of cervix relatively more than the other two sites. The study showed that the position and the fixation of the folly catheter in the last $3^{\text {rd }}$ of the cervical canal and toward the utero-cervical opening gave more number of embryos than the two other sites upon the process of flushing and collection of embryos.

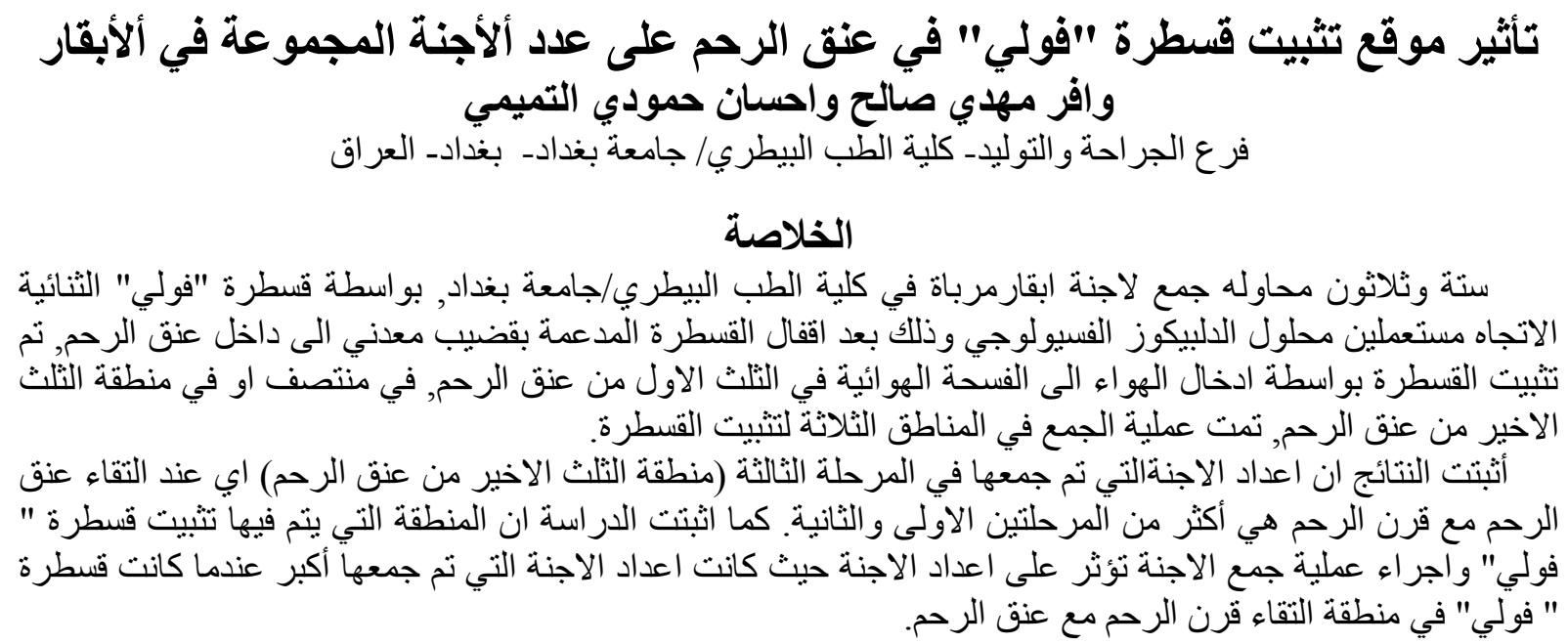

\section{Introduction}

Since the first trails of Embryo Transfer Technique has done by (1) and those done by (2) on bovine to have a successful transplant, especially when those techniques played important tools in the improvement of livestock populations, scientists concentrated their research works to modulate facilities to better good results. The superovulation, synchronization, donor animal selection, changing from surgical embryo collection to non-surgical method (3), flushing media, surgical and non-surgical embryo transfer (4) and the ways of evaluations of collected embryos are those facilities in this technique.

All those previous items played effective attempts on the final results to obtain progressive number of embryos upon detection, and each one may have its important effects on the results, but as we working with this technique, the effects of collections of embryo may have a little bit more importance than the others (5). As the non-surgical method of collection gave more accurate and quiet easy application due to less risk on the donor animal and can be repeated more than one time on the same donor (6), especially if we were applying it on a valuable donor (7), together with the previous causes, this method can be accomplished in the field with a simple preparations. The non-surgical method of collection done by two ways foley catheter introduced in the donor's cervix, and by air inflated air bubble to fix the catheter in the cervix, via flushing the collecting media undergo detection by the stereomicroscope to search for embryos. Beside that and due to the bovine cervical anatomy, the 
cervix of the cow has a kind of CRIPTS which give the cervix a ridged texture (8), those CRIPTS were related to the presence of transverse or spiral interlocking ridges which are known as the annular rings (usually 4-5) which give the cervix a thick wall and constricted lumen (9). The nature of this part due to that complex anatomy could be affected the passage of the catheter and may be interfere with the fixation of it.

The presence of those CRIPTS gave the cervical passage a chance to trap any objective materials from getting in or out the uterus as well as embryos (10). Describe the effects of the deferent sites of fixation of the end of Foley catheter in the cervix and show that as the fixation sites toward the utero-cervical junction give more flushed embryos than any other sites, and this is mainly due to the complexity of the anatomy of the bovine cervix.

\section{Materials and Methods}

The study was designed in two applications:

1. Laboratory applications using Fresh genitalia specimens:

A. The normal bovine genital system before the introduction of the Foley catheter (Twoways Foley catheter size 20, $30 \mathrm{ml}$ Maersk Medical A/S Engmosenl. 3540 Lynge-Denmark) in the cervix shown in figure (1A)

B. The bovine genitalia where the Foley catheter fixed at the first third of the cervical canal figure (1B).

C. The bovine genitalia where the Foley catheter fixed at the middle of the cervical canal figure (1C).

D. The bovine genitalia where the Foley catheter fixed at the last third of the cervical canal and near the utero-cervical junction figure (1D).
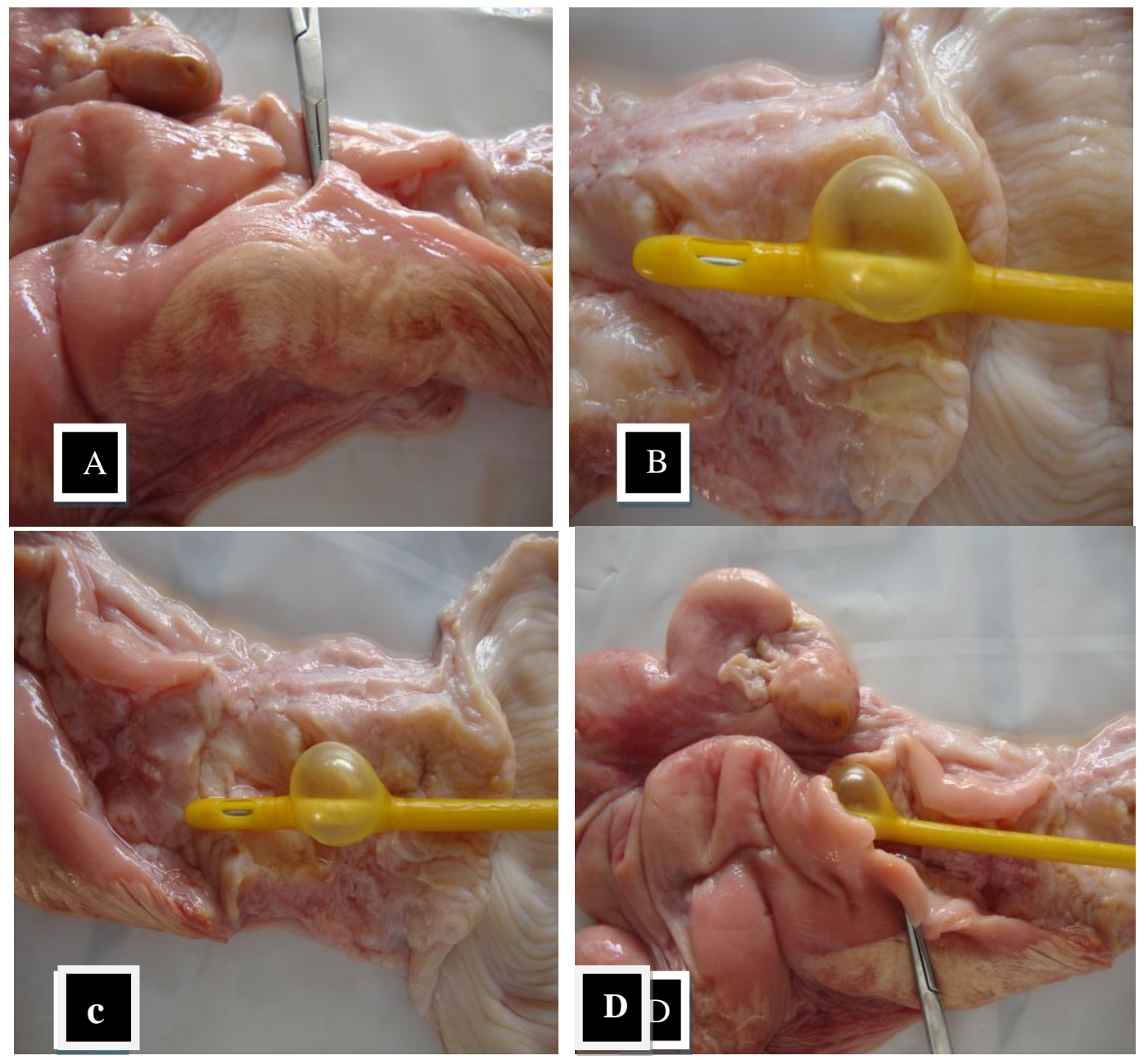

Figure 1: Show normal cow genetalia: before the introduction of the Foley catheter in the cervix (A), after introduction of Foley catheter in the first third (B), at the middle (C) or at the last third of the cervical canal (D). 


\section{Proceeding of the Eleventh Veterinary Scientific Conference, 2012; 232-236}

\section{2- Field applications:}

A. Preparation of the donors: All donors were already Super ovulated on day $10^{\text {th }}$ of the cycle $(\mathrm{E}=0)$ by one injection of (3500 IU) Folligon ${ }^{\circledR}$ (PMSG- Intervet International B.V. Boxmeer-Holland) and on day $12^{\text {th }}$ of the cycle received $2 \mathrm{ml}$ of Prosolvin ${ }^{\circledR}$ (LoprosteolIntervet International B.V. Boxmeer-Holland) and inseminated artificially as the signs of estrus appeared.

B. Flushing methods: All donors (at day 6 from the insemination time) will be prepared for the flushing as gave them a low epidural nerve block (Lignocaine ${ }^{\circledR} 2 \%$ V.M.D. NV, HOGE MAUW900, 2370 ARENDONK, BELGIUM), the external genitalia washed carefully and the cervix grasped and fixed by one hand of the veterinarian through the rectum, by the assistant hand the two vulvar lips slightly apart, the Foley catheter Extended by the metal robe slightly and carefully introduced via the vagina to the cervix. The catheter will be introduced at the beginning of the cervical canal or at the First $3^{\text {rd }}$ and by air inflation using a plastic syringe (about $10-15 \mathrm{ml}$ of air will be pushed in the air bubble) to fix the catheter, after that, the veterinarian try to push the catheter slightly backward to be sure about that fixation. About 80-100ml of the flushing media (Flushing media Immunosystem- 311 McKay Ave Sprig Valley- WI 54767 USA) will be pushed to flush the two uterine horns together, these steps will be repeated 12 times, the veterinarian examined the ovaries of each donor to be sure about the number of corpora lutea found on the 2 ovaries, the flushing media will be examined for embryos detection and the result was recorded. The $2^{\text {nd }}$ trail were accomplished as the end of the foley catheter near the middle of the cervical canal, and by inflation the air bubble the end of the Foley catheter is fixed, the process of flushing will be carried out for 12 attempts as in the previous method, then the other steps of the work carried as in the $1^{\text {st }}$ trial. The $3^{\text {rd }}$ trail where the catheter was pushed slightly forward to fix near the Last third of the cervical canal and near the utero-cervical junction of the left horn first where the fixation site does, and by air bubble inflation, the end of the catheter will be inside the horn, the flushing achieved by pushing about $60 \mathrm{ml}$ inside the horn and these steps last for six times before changing the site of the catheter to the other horn to be flushed individually, then the flushing media For both horns will be examined and then the other steps of the work carried as in the $1^{\text {st }}$ and the $3^{\text {rd }}$ trails. All results will be recorded.

\section{Results}

A. Low Epidural Nerve block:- All animals included in this study showed variable response to the Lignocaine $2 \%$, started from dose of $7 \mathrm{ml}$ injected in the space between the $1^{\text {st }}$ and the $2^{\text {nd }}$ coccygeal vertebrae to a dose of $10 \mathrm{ml}$ as we started at first by dose of $5 \mathrm{ml}$ and then we elevated the dosing gradually until we have the relaxation response. The signs of response we considered positive was the tail relaxation and loss of sensation via nail sting.

B. Examination of the genitalia:

All the donors occupied in this study showed slightly the nearest pictures of the status of their genitalia via rectal examination including position and texture of the cervix, situation of the horns, ovaries which are the main organ that the focusing of the examination directed, show some degree of variations according to the increasing in size, more prominent and having about 2-6 corpora lutea in each one.

C. The intromission of the Foley catheter:

All the donors of this study and even with a good results obtained by the low epidural nerve block showed a kind of not comfortable manners as we tried to push and introduced the foley catheter through the cervix mainly with the animals of the $2^{\text {nd }}$ and $3^{\text {rd }}$ groups, but once the catheter passing through and fixed by the air bubble and the flushing methods carried out they stayed calm and quiet. Animals of the $3^{\text {rd }}$ group appeared to need more air inflation to fill-up the air bubble upon the fixation step as the end of the catheter by the utero-cervical junction. The fixation sites of all groups after air inflated appeared as good as enough to make the flushing methods run smoothly by pulling the catheter gently backward after that fixation.

D. The flushing technique: 1- First group: The first group where the end of the Foley catheter fixation was at the $1^{\text {st }}$ third of the cervical canal, noticing of that the quantity of the flushing 


\section{Proceeding of the Eleventh Veterinary Scientific Conference, 2012; 232-236}

media is about $80-100 \mathrm{ml}$ in each flushing which was mainly enough to flush the two horns felt by the veterinarian hand that grasped the cervix, and for the 12 attempts the only 2 embryos detected in the flushing media which were of early blastocyst Table (1B).

1. Second group: The second group where the end of the Foley catheter fixation was at the middle portion of the cervical canal, the flushing media needed in each one was about 80-100 $\mathrm{ml}$ to flush the both horns .Two embryos were detected after the examination of the flushing media, one of them of a tight morulla and the other one was of blastocyst (table 1).

2. Third group: The third group where the end of the Foley catheter fixation near the uterocervical junction and mainly toward the last third of the cervical canal the pushing of the end of the catheter was done firmly and the entrance to the beginning of the horn was finely smooth, guided by the other hand inside the rectum to fixed in the left horn, air inflation about 20-25 ml slightly more than the above two groups, and the flushing media about $45-60 \mathrm{ml}$ in each 6 trail, then we did it as the same to the right horn and also for 6 trails. The result of examination of the flushing media detected 5 embryos, 2 as blastocyst and 3 as morulla (table $1)$.

Table (1): Number of corpora lutea, and the number of embryos collected by foley catheter fixed at different sites in the cervix of cows.

\begin{tabular}{|c|c|c|c|c|c|c|c|}
\hline \multirow[b]{2}{*}{ group } & \multirow{2}{*}{$\begin{array}{l}\text { No. of } \\
\text { CL/cow } \\
\text { (mean) }\end{array}$} & \multirow{2}{*}{$\begin{array}{l}\text { Total volume (L) } \\
\text { of } \quad \text { media } \\
\text { used/cow }\end{array}$} & \multirow{2}{*}{$\begin{array}{l}\text { No. of } \\
\text { trials }\end{array}$} & \multicolumn{4}{|c|}{ No. of embryos collected/cow (mean) } \\
\hline & & & & blastocyst & morulla & Total & $\begin{array}{l}\text { Collected } \\
\text { embryos/CL (\%) }\end{array}$ \\
\hline $\mathbf{1}^{\mathrm{st}}$ & 4 & 1 & 12 & 2 & - & 2 & $50 \%$ \\
\hline $\mathbf{2}^{\text {nd }}$ & 3.5 & 1 & 12 & 1 & 1 & 2 & $58 \%$ \\
\hline $3^{\text {rd }}$ & 6 & 0.3 & 12 & 2 & 3 & 5 & $84 \%$ \\
\hline
\end{tabular}

\section{Discussion}

A. The animals of this study showed variable response to the Lignocaine, this variation of the effects of the low epidural nerve block depending on many factors such as the weight of the animals, the space of the dural sac and may be due to some kind of individual variations (11). B. Examination of the genitalia:

The ovaries was appeared in this study with variables response to the effects of the PMSG, as shown with the number of the corpora lutea and the increasing in size (12) described these responses especially with the usage of PMSG and also mentioned by (13) when study the effects of the hormonal dosage on the ovarian response.

C. The flushing technique:

The study showed that the sites of the fixation of the Foley catheter has a direct effect on the number of the embryos, and this is mainly due to the anatomy of the bovine cervical canal and because to the presence of the CRIPTS which is in a way they trapped some of the flushed embryos in between. Many authors showed that the percentage of flushed embryos increased when the end of the Foley catheter was fixed near the utero-cervical junction (14), this is also reported by (10) who described deferent fixation sites and mentioned that the fixation near the last third of the cervical canal give more number of embryos, also (15) obtained more embryos as the end of the catheter pushed forward and beyond the end of the cervical canal and at beginning of the uterine horn. This study will simply give a valuable result for those who want to work in the field of the embryo transfer by lead them to fact that many factors will interfere with their work and directly mimic the end results, one of those is the fixation of the end of Foley catheter and as the end is near the utero-cervical junction the final result is better and the number of embryos collected will be elevated.

\section{References}

1- Heape, W. (1890). Preliminary note on the transplantation and growth of mammalian ova within uterine foster-mother. Proc. soc. London Vol.48 p.457-458, cited by Robert, S. J. Veterinary Obstetrics and genital diseases (1986) 
2- Willett, E. L., Black, W.G., Casida, L. E., Stone, W. H. and Bunker, P.J. (1951). Successful transplantation of bovine ova. Science N.Y. vol.113 p.747.

3- Sreenan, M. J. (1978). Non-surgical egg recovery and transfer in the cow. Vet. Rec. vol.102 p.58-60.

4- Thompson, C.K. and Fogwell, R. L. (1982) A Paracervical method for non-surgical Transfer of bovine embryos. J. of Therio.,18 (6): 629-632.

5- Shelton, J.N., Helth, T. D., Old,K. G. and Turnbull, S. J. (1979) Non-surgical recovery Of egg from single ovulating bovine. J. of Therio. 11(2 ):149-151.

6- Morrow, D. A. (1980) Current therapy in theriogenology. Cited by Hafez E.S.E. reproduction in farm animals. $5^{\text {th }}$ ed. LEA and FEBIUER-Philadelphia.

7- Oguri, N. and Tsutsumi, Y. (1982) Non-surgical transfer of equine embryo. In Vitro fertilization and embryo transfer. ESE Hafez and K. Semm (eds.) Lancaster. England. MTP Press Limited.

8- Hunter, R. H. F. (1982) Reproduction in farm animal. London. Longman, p56-57.

9- Sisson, S. B. (1972) The Anatomy of the Domestic Animals .W.B. Saunders company, p 616-617.

10- Schneider, U. and Hann, J. (1979) Bovine embryo transfer in Germany. J. of Therio. Vol. 18 No. 4 p. 413-422

11- Tufvesson, G. (1963) Local Anesthesia in Veterinary Medicine. Stockholm, p40-41.

12- Elsden, R. P. and Seidle, G. E. (1985) Procedures for recovery, bisection, freezing And transfer of bovine embryo. Animal reproduction lab. Colorado state university, Fort Collins.

13- Mihm, M., Deletang, F. and Roche, JF. (1998). The gonadotropins and ovarian response to an intermediate or low dose of gonadorelin in beef heifers: influence of dose, follicle status and progesterone environment. j. of Repro. and fertility Abstr. 21:74.

14- Newcomb, R., Christie, W.B. and Rowson, L.E. (1978) Non-surgical recovery of bovine embryo. Vet. Rec., 102:44.

15- Elsden, R.P. (1987) Manual for embryo transfer. J. of Society for theriogenology, p4-5. 